\title{
Thomas Spranz-Fogasy
}

\section{Argumentation als alltagsweltliche Kommunikationsideologie}

In den vergangenen zweieinhalb Jahrtausenden hat sich die Argumentation in der westlichen Welt zu dem zentralen Verfahren gewaltfreier Problemund Konfliktlösung entwickelt. Argumentation als Verfahren scheint den Menschen Kriterien und Möglichkeiten eines rationalen Diskurses zu versprechen. Diese so selbstverständliche Annahme wurde aber nie daraufhin geprüft, ob sie mit den Bedingungen und Zwängen der Durchführung von Gesprächen vereinbar ist.

Auf der Basis einer detaillierten linguistischen Gesprächsanalyse von mehr als 60 alltagsweltlichen Problem- und Konfliktgesprächen wurde untersucht, wie Argumentation in Gesprächen aussieht und wie sie funktioniert. Dabei zeigt sich, dass Argumentation ein Hilfsmittel für Gesprächsteilnehmer ist, strittige oder unklare Darstellungen gesprächslokal zu behandeln, ohne dabei den übergeordneten Handlungsfaden aufzugeben. Die Ergebnisse einer Argumentation werden dann wieder zurückgebunden und zur weiteren Bearbeitung übergeordneter Handlungsaufgaben des Gesprächs genutzt.

Hinsichtlich der Beschreibung von Argumentation in Gesprächen lässt sich zweierlei unterscheiden, die Argumentationsstruktur auf der einen und die inhaltlichen Aktivitäten auf der anderen Seite. Strukturell lassen sich fünf Sequenzschritte bestimmen, in denen Argumentationen gesprächsweise entfaltet werden: (1) eine Argumentation auslösende Handlung, (2) eine Widerspruchsbzw. Problematisierungshandlung, (3) eine Darlegungs- oder Begründungshandlung, (4) die Akzeptanz einer Position und schließlich (5) deren Ratifikation. Diese Sequenz kann nun beliebig erweitert werden durch Insertion weiterer Problematisierungen und Begründungen, sie kann aber auch extrem kondensiert werden bis hin zu einer Parenthese innerhalb eines laufenden Beitrags. Und natürlich kommt es empirisch häufig vor, dass die Schritte Akzeptanz und Ratifikation ersetzt werden müssen durch beispielsweise Vertagung, Feststellen eines bleibenden Dissens oder andere Verfahren, die aber in der Regel ebenfalls eine Rückkehr ins übergeordnete Handlungsgeschehen ermöglichen. In inhaltlicher Hinsicht lassen sich dann aus den Aktivitäten von Gesprächsteilnehmern verschiedene Kriterien ermitteln, die sie selbst als Kriterien ihres Argumentierens 
kennzeichnen und die sie in gesprächsrhetorische Verfahren umsetzen: Sie beanspruchen damit beispielsweise Faktizität bei eigenen Behauptungen, sie kritisieren mangelnde Relevanz in Äußerungen der Gesprächspartner oder sie demonstrieren die Stimmigkeit und Kohärenz ihrer eigenen Äußerungen.

Interaktive Sequenzierung und inhaltliche Bezüge machen nun deutlich, dass Gesprächsteilnehmer dabei auf interaktionskonstitutive Elemente abheben: Was zur Herstellung von Gesprächen notwendig ist - wie beispielsweise zur Sache zu sprechen oder den Partner ausreden zu lassen -, wird in Gesprächen in Argumente umgemünzt.

In dieser Perspektive ist Argumentation als eine soziale Handlungspraxis bestimmt, deren Ursprung in den Bedingungen, Möglichkeiten und Zwängen von Gesprächen, von sozialer Interaktion überhaupt liegt. Argumentativ erfolgreich kann nur sein, wer seine Aussagen als handlungsrelevant, seine Person als integer und kompetent, und dann auch seine Aussagen als stimmig, angemessen und stichhaltig durchsetzt. Interaktiv gültig ist letztlich nur, was Gesprächsteilnehmer in Folge ihrer Argumentation akzeptieren. Die für Argumentation notwendige und konstitutive Anbindung an übergeordnete Handlungsorientierungen widerspricht dabei fundamental der Idee einer rein sachbezogenen und von Interessen unbeleckten Aushandlung, wie sie seit der Antike in den Wissenschaften, aber auch im Alltagsdenken der Gesellschaftsmitglieder vorherrscht.

Was Gesprächsteilnehmer dabei antreibt, ist die Kraft des intersubjektiven Glaubens an Argumentation als ein Verfahren zur Entwicklung einer gemeinsam geteilten Perspektive; und der Anspruch an das Verfahren als ein Validität garantierendes Verfahren wird dabei außerdem noch mit dem Anspruch auf die Validität des Ergebnisses einer Argumentation verwechselt. Die Kraft des intersubjektiven Glaubens und der Anspruch an das Verfahren sind die zentralen Bestandteile dessen, was als Kommunikationsideologie bezeichnet werden kann.

Diese Einsicht ist nun aber kein Anlass zur allgemeinen Depression oder zum Verzicht auf Argumentation als Verfahren der Erkenntnis: Strittiges oder Unklares zwischen Gesellschaftsmitgliedern wird es immer geben - wie schon die Unterschiedlichkeit der Individuen als basale Voraussetzung sozialer Interaktion vedeutlicht. Als soziale Handlungspraxis zwingt Argumentation aber dazu, die Ansprüche an Äußerungen im offenen sozialen Feld zu verhandeln, sie zur Disposition zu stellen, mit Widerstand umzugehen und dann neu zu formulieren. Dabei müssen komplexe Sachverhalte differenziert werden, und es 
werden neue Zusammenhänge und neue Konfigurationen sichtbar - Argumentation ist in dieser Hinsicht eine ausgesprochen produktive Kommunikationsform mit einem erheblichen Potenzial für Prozesse der gesellschaftlichen Entwicklung.

\section{Literatur}

Spranz-Fogasy, Th. (1999): Interactional Resources of Argumentation. In: van Eemeren, F.H./ Grootendorst, R. u. a. (Hrsg.): Proceedings of the Fourth International Conference of the International Society for the Study of Argumentation (University of Amsterdam, 16-19, 1998). Amsterdam, S. 761-763

Spranz-Fogasy, Th. (2002): Interaktionsorganisation als (meta-)kommunikative Ressource des Argumentierens. In: Bastian, Sabine/Hammer, Francoise (Hrsg.): Argumentation und Metakommunikation. Frankfurt, S. 11-25

Spranz-Fogasy, Th. (2002): Alles Argumentieren oder was? - Zur Konstitution von Argumentation in Gesprächen. In: Deppermann, A./Hartung, M. (Hrsg.): Argumentieren im Gespräch (Arbeitstitel). Tübingen

Spranz-Fogasy, Th. (2002): Argumentative sequencing and its interactional variation. In: Meierkord, C./Fetzer, A. (Hrsg.): Rethinking Sequentiality (Arbeitstitel). Amsterdam 